\title{
Burnout or not? Is Sudoku really that stressful?
}

\author{
Stojanovic Stipic S. ${ }^{1}$, Babacanli A. ${ }^{2}$, Baric A. ${ }^{3}$, Pavicic Perkovic ${ }^{1}$, Dropulic N. ${ }^{1}$, Stipic T. ${ }^{4}$
}

\author{
${ }^{1}$ Department of Anaesthesiology \& Intensive Care, Split, Croatia, \\ ${ }^{2}$ Department of Emergency Medicine - Zagreb (Croatia), \\ ${ }^{3}$ Department of Anaesthesiology and Intensive Care Medicine - Zagreb (Croatia), \\ ${ }^{4}$ Department of Dermatology - Split (Croatia)
}

\section{Background and Goal of Study:}

Considering anesthesiologists among other medical specialties, it is well known that they have an increased risk of suffering from burnout syndrome. Unfortunately, burnout syndrome is often related to lower work performance, coronary artery disease and deteriorating overall mental health, all of which may seriously affect the patient's treatment.

The aim of this paper is to compare possible burnout syndrome among Croatian anesthesiologists.

\section{Materials and Methods:}

This research involved 2568 doctors working in different fields of medicine including 177 anaesthesiologists. The Maslach Burnout Inventory (MBI) was used to measure burnout. MBI defines burnout as a three-dimensional syndrome via 22 questions; 9 question relate to emotional exhaustion (EE), 5 question relate to depersonalization (DP) and 8 question relate to diminished personal accomplishment (PA). Possible answers on the main scale ranged from $0=$ never to $6=$ every day. We created 3 subscales for every MBI dimension; EE subscale with severity indeks (SI) ranging from $\geq 27$ for high, 27-16 for moderate and $\leq 16$ for low, DP subscale with $\mathrm{SI} \geq 13$ for high, 13- 6 for moderate and $\leq 6$ for low and PA subscale with SI $\geq 39$ for high, 39-31 for average and $\leq 31$ for low.
Results and discussion:

All 2568 doctors had EE of 30\% (high), DP of $8,75 \%$ (moderate) and PA of 29,9\% (low). 63\% of anesthesiologist showed high EE, 57\% exibited high PA and 46\% had low DP. Emotional exhaustion is lowest when their career begins or ends.

Depersonalization deteriorates over time whereas diminished personal accomplishment is constant throughout the working years. We also found positive correlation between DP total score and years of experience $(r=-0,320, p<0,0001)$. A total of $55(33 \%)$ were resident, $72(42 \%)$ consultants and 44 $(25 \%)$ subspecialist and we found statistical significant differences of DP score $(\mathrm{p}=0,026)$.

\section{Conclusion(s):}

Anestesiologists are not trained to fail. In relation to sense of increased responsibility and perfectionism, these factors trigger burnout syndrome. It often emerges when there is insufficient workforce so fewer anesthesiologists must compensate, often working overtime, for the lack of their colleagues. Results show that prevention of burnout is highly necessary among Croatian anesthesiologists and it involves a spectrum of measures, ranging from changing the working conditions to taking care of employee's overall mental health.

\begin{tabular}{lll}
\hline \multicolumn{1}{c}{ EE } & Consultants & Resident \\
\hline High & 51 & 30 \\
& $70.8 \% \mathrm{CT}$ & $54.5 \% \mathrm{CT}$ \\
& $29.8 \% \mathrm{GT}$ & $17.5 \% \mathrm{GT}$ \\
Low & 14 & 7 \\
& $19.4 \% \mathrm{CT}$ & $12.7 \% \mathrm{CT}$ \\
Moderate & $8.2 \% \mathrm{GT}$ & $4.1 \% \mathrm{GT}$ \\
& 7 & 18 \\
& $9.7 \% \mathrm{CT}$ & $32.7 \% \mathrm{CT}$ \\
& $4.1 \% \mathrm{GT}$ & $10.5 \% \mathrm{GT}$ \\
& 72 & 55 \\
& $(42.1 \%)$ & $(32.2 \%)$ \\
\hline
\end{tabular}

\begin{tabular}{cllll} 
PA & Consultants & Resident & \multicolumn{2}{c}{ Subspecialist } \\
High & 43 & 25 & 29 & $97(56.7 \%)$ \\
& $59.7 \% \mathrm{CT}$ & $45.5 \% \mathrm{CT}$ & $65.9 \% \mathrm{CT}$ & \\
& $25.1 \% \mathrm{GT}$ & $14.6 \% \mathrm{GT}$ & $17.0 \% \mathrm{GT}$ & \\
ow & 8 & 6 & 5 & $19(11.1 \%)$ \\
& $1.1 \% \mathrm{CT}$ & $10.9 \% \mathrm{CT}$ & $11.4 \% \mathrm{CT}$ & \\
& $4.7 \% \mathrm{GT}$ & $3.5 \% \mathrm{GT}$ & $2.9 \% \mathrm{GT}$ & \\
Moderate & 21 & 24 & 10 & $55(32.2 \%)$ \\
& $29.2 \% \mathrm{CT}$ & $43.6 \% \mathrm{CT}$ & $22.7 \% \mathrm{CT}$ & \\
& $12.3 \% \mathrm{GT}$ & $14.0 \% \mathrm{GT}$ & $5.8 \% \mathrm{GT}$ & \\
& 72 & 55 & 44 & 171 \\
& $(42.1 \%)$ & $(32.2 \%)$ & $(25.7 \%)$ & \\
& & & &
\end{tabular}

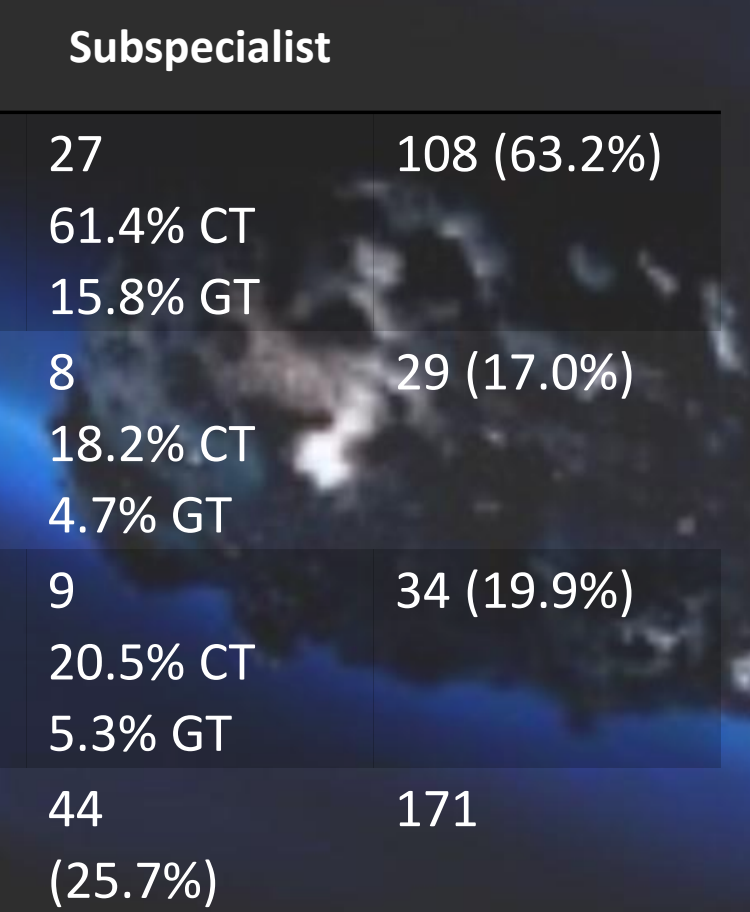

\begin{tabular}{ll}
\hline \multicolumn{1}{c}{ DP } & Consultants \\
\hline High & 17 \\
& $3.6 \% \mathrm{CT}$ \\
& $9.9 \% \mathrm{GT}$ \\
Low & 39 \\
& $54.2 \% \mathrm{CT}$ \\
& $22.8 \% \mathrm{GT}$ \\
Moderate & 16 \\
& $22.2 \% \mathrm{CT}$ \\
& $9.4 \% \mathrm{GT}$ \\
& 72 \\
& $(42.1 \%)$ \\
\hline
\end{tabular}

Resident
21
$38.2 \%$ CT
$12.3 \% \mathrm{GT}$
16
$29.1 \% \mathrm{CT}$
$9.4 \% \mathrm{GT}$
18
$32.7 \% \mathrm{CT}$
$10.5 \% \mathrm{GT}$
55
$(32.2 \%)$

Subspecialist 13.6\% CT $3.5 \%$ GT 24 $54.5 \%$ CT $14.0 \%$ GT 14 $31.8 \%$ CT $8.2 \%$ GT 44 (25.7\%)
$44(25.7 \%)$

79 (46.2\%)

$48(28.1 \%)$

171 